\title{
Propagation of obstructed Bessel and Bessel-Gauss beams
}

\author{
Igor A. Litvin ${ }^{\mathrm{a}, \mathrm{b} \dagger}$, Melanie G. McLaren ${ }^{\mathrm{a}, \mathrm{c}}$ and Andrew Forbes ${ }^{\mathrm{a}, \mathrm{d}:}$ \\ ${ }^{a}$ CSIR National Laser Centre, PO Box 395, Pretoria 0001, South Africa \\ ${ }^{\mathrm{b}}$ Laser Research Institute, University of Stellenbosch, Stellenbosch 7602, South Africa \\ ${ }^{\mathrm{c}}$ School of Physics, University of the Witwatersrand, Private Bag 3, PO Wits 2050, Johannesburg, \\ South Africa \\ ${ }^{\mathrm{d}}$ School of Physics, University of KwaZulu-Natal, Private Bag X54001, Durban 4000, South Africa
}

\begin{abstract}
The investigation into Bessel beams has been a topic of immense research during the past 20 years, due to the interesting properties they display. Bessel beams not only exhibit diffraction free propagation, but also reconstruction of the amplitude and phase of the beam after encountering an obstruction. Although this self reconstruction property has been previously modelled by numerous groups, the techniques involve rigorous, time-consuming computations. In this work we present an efficient method to accurately calculate the reconstruction of a Bessel beam after an arbitrary obstruction. Our method considers the well-known conical wave features of Bessel beams and looks at the projection of the obstruction in space as a result of the travelling conical waves that produce the Bessel beams.
\end{abstract}

Keywords: Bessel-Gauss beams; reconstruction; conical waves.

\section{INTRODUCTION}

Bessel beams (BBs) represent a class of so-called diffraction free solutions to the Helmholtz equation, and have been studied extensively since the seminal work of Durnin et al. ${ }^{1}$ in the late 1980s. One of the simplest solutions illustrated by Durnin ${ }^{2}$, describes a monochromatic wave with an electric field envelope that is a zeroth-order Bessel function of the first kind, given by $J_{0}\left(k_{r} r\right)$, where $k_{r}$ is the radial component of the wave vector $k$ and $r$ denotes the distance from the zaxis. This was the first mathematical concept of the Bessel beam, which ideally consists of an infinite number of rings of light, carrying an infinite amount of power over an infinite area. Experimentally, it is not possible to generate such a beam, and so an approximation is made in the form of a Bessel function enveloped by a Gaussian profile, thereby limiting the energy carried by the field to some finite value.

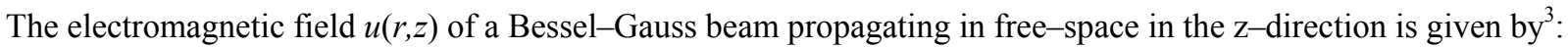

$$
\begin{aligned}
u(r, z) & =A \frac{\omega_{0}}{\omega} \exp \left[i\left(k-\frac{k_{r}^{2}}{2 k}\right) z-i \Phi(z)\right] J_{0}\left[k_{r} r /\left(1+i z / z_{r}\right)\right] \\
& \times \exp \left[\left(\frac{-1}{\omega^{2}(z)}+\frac{i k}{2 R(z)}\right)\left(r^{2}+\frac{k_{r}{ }^{2} z^{2}}{k^{2}}\right)\right]
\end{aligned}
$$

where $A$ is an amplitude factor, which may be complex, and

$z_{r}=\pi \omega_{0}^{2} / \lambda$

${ }^{\dagger}$ Corresponding author: ilitvin@csir.co.za

* Corresponding author: aforbes1@csir.co.za 


$$
\begin{aligned}
& \omega(z)=\omega_{0} \sqrt{1+\left(z / z_{r}\right)^{2}} \\
& R(z)=z\left[1+\left(z_{r} / z\right)^{2}\right] \\
& \Phi(z)=\tan ^{-1}\left(z / z_{r}\right)
\end{aligned}
$$

These equations represent the Rayleigh length, the beam width, the radius of curvature of the wavefronts and the Guoy phase of a Gaussian beam respectively. The Rayleigh length determines the distance over which a Gaussian beam can propagate without diverging significantly and depends on the beam width at the waist, $\omega_{0}$, and the wavelength of the beam, $\lambda$.

These "pseudo non-diffracting" beams maintain the properties associated with ideal Bessel beams, but only over a finite distance. In particular, they satisfy that the beam be propagation-invariant and also display the interesting property of reconstruction. The latter property has been used in a variety of applications, including optical tweezing ${ }^{4}$ and medical imaging $^{5}$, and is therefore an intriguing property worth investigating. Reconstruction has been well explained by firstly considering a Bessel beam as a superposition of plane waves, whose transverse amplitudes reform after encountering an obstruction $^{6}$. Theoretical studies of this property describe reconstruction under varying conditions, including in a nonlinear medium ${ }^{7}$ and by wave packets due to spatial-temporal links ${ }^{8}$. The reliability of the periodical selfreconstruction, as well as the wavelength dependence of white light Bessel beams have been examined ${ }^{9}$. Experimentally, reconstruction of Bessel beams has also been verified on numerous occasions by means of different methods.

Although extensive work has been devoted to this one property, the methods of modelling this characteristic are limited in accuracy and speed, and are yet to calculate Bessel fields after arbitrary obstructions. Conventionally, the FresnelKirchoff diffraction integral together with Babinet's principle has been employed, which results in time consuming calculations. Recently, the phenomenon of reconstruction was eloquently explained by considering the dynamics of the conical waves that form a Bessel beam ${ }^{10}$. Essentially, the cone of wave vectors previously mentioned consists simultaneously of two conical waves, an incoming and an outgoing wave, which can be represented by Hankel functions ${ }^{11}$ of the second, $H_{0}^{(2)}\left(k_{r} r\right)$, and first, $H_{0}^{(1)}\left(k_{r} r\right)$, kind respectively:

$$
\begin{aligned}
& H_{0}^{(1)}\left(k_{r} r\right) \exp \left(i k_{z} z\right)=\left[J_{0}\left(k_{r} r\right)+i N\left(k_{r} r\right)\right] \exp \left(i k_{z} z\right) \\
& H_{0}^{(2)}\left(k_{r} r\right) \exp \left(i k_{z} z\right)=\left[J_{0}\left(k_{r} r\right)-i N\left(k_{r} r\right)\right] \exp \left(i k_{z} z\right),
\end{aligned}
$$

where $N\left(k_{r} r\right)$ is the zero-order Newman function.

The incoming conical wave transforms into the outgoing conical wave and the superposition of these two conical waves is crucial in obtaining propagation invariant beams. Within a Bessel beam, although locally the light travels parallel to the axis of propagation, the energy flux follows the direction determined by the cone of wave vectors. For this reason, reconstruction after an obstruction is observed. That is, the reconstructed beam results from light that was not obscured by the obstacle.

Despite this fluent explanation by Anguiano-Morales et al. ${ }^{10}$, the numerical simulations demonstrated resorted back to solving the Helmholtz equation. In this paper we present an efficient and accurate technique to predict Bessel and Bessel-Gauss beam propagation after encountering an obstruction of arbitrary geometry and complex orientation (i.e., no symmetry is required in the obstacle under study). The technique is a simple exploitation of the well-known conical wave formalism of Bessel beams, and is based on a projection of the obstacle in space due to the travelling conical waves that form the Bessel beams. We verify the concept experimentally for two obstacle geometries, and show that despite the method being based on a ray optics approximation, the method is resilient even when diffraction is evident. 


\subsection{Fundamentals of Bessel beams}

The propagation and generation of Bessel-Gauss (BG) beams have been well documented ${ }^{3,12,13}$. Equation (1) can be simplified by considering the field at the plane $\mathrm{z}=0$, where the intensity profile is shown in Fig. 1 (a):

$$
u(r, 0)=A J_{0}\left(k_{r} r\right) \exp \left[-\left(r / \omega_{0}\right)^{2}\right]
$$

Fig. 1 illustrates how the intensity profile of Eq. (1) changes along the axis of propagation (z-direction), from a Bessel beam (a) to an annular ring (b).

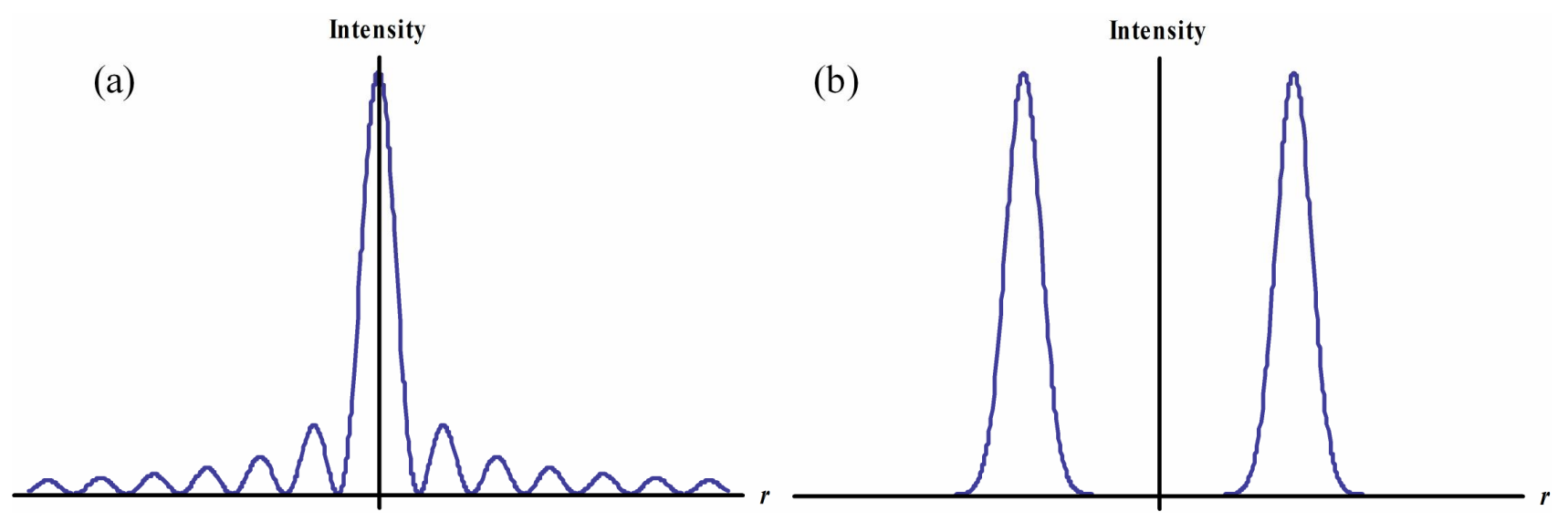

Fig. 1: Intensity profiles of a Bessel-Gauss beam. (a) A Bessel beam profile is produced near $z=0$, (b) while an annular ring is produced at larger propagation distances.

An important property of the Bessel beam is its non-diffracting nature. That is, for propagation in the $z$ direction, the intensity satisfies,

$$
I(x, y, z \geq 0)=I(x, y)
$$

where $I(x, y, z) \propto J_{0}^{2}\left(k_{r} r\right)$ for a zero-order Bessel beam.

Of course, for a BG beam this equality is true only for a finite distance of $z$. This distance can be calculated by analysing the experimental generation of a BG beam. There are two well established methods for Bessel beam formation, one of which makes use of an annular slit. A Bessel beam can be thought of as a superposition of plane waves whose wave vectors lie on a cone, which in $k$-space reveals itself as a ring. That is, the Fourier transform of a ring results in a Bessel beam, which allowed Durnin et al. ${ }^{1}$ to produce an approximation to a Bessel beam by illuminating an annular slit onto the back focal plane of a focusing lens. A more efficient method of Bessel beam generation makes use of an axicon. Fig. 2 illustrates how a Bessel beam is created by illuminating a Gaussian beam onto an axicon. The opening angle of the cone is given by,

$$
\theta=(n-1) \gamma
$$

where $n$ is the refractive index of the axicon and $\gamma$ is the opening angle of the axicon. 


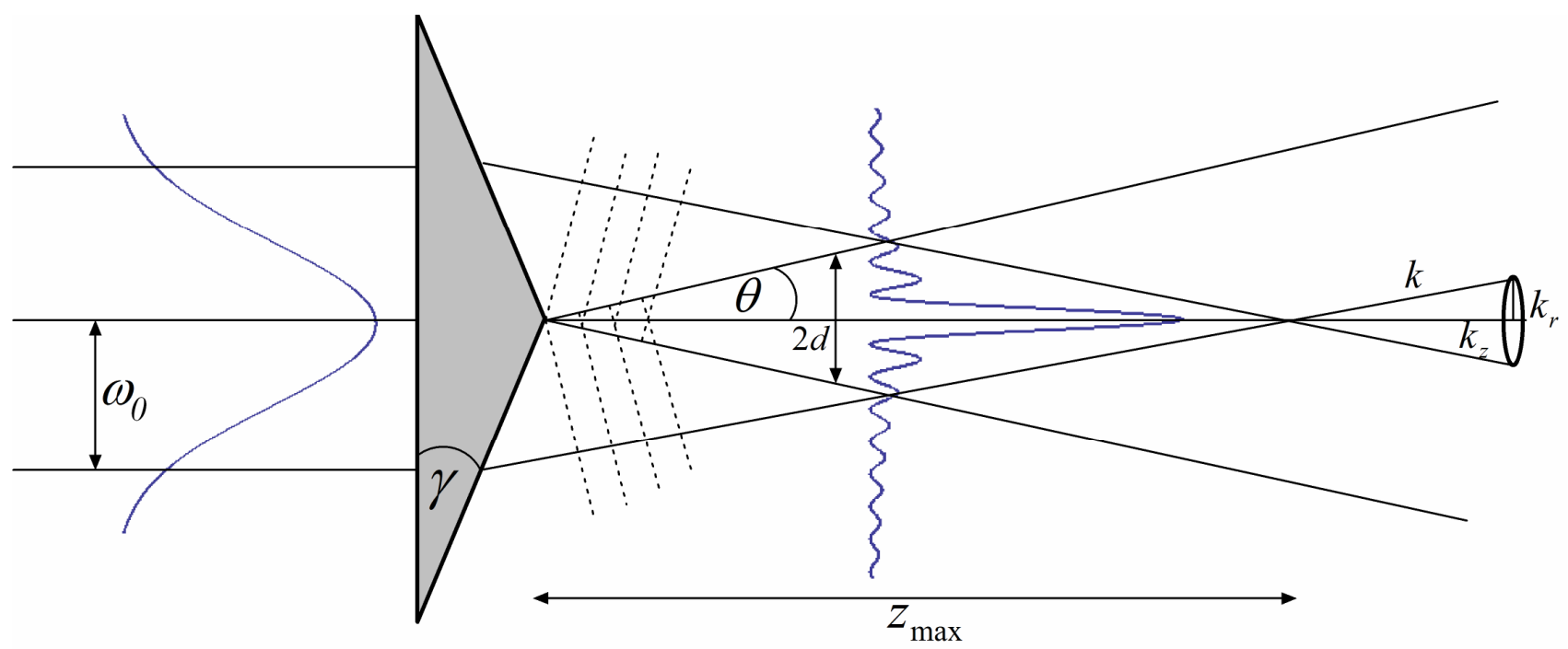

Fig. 2: Diagram illustrating the finite propagation distance of a Bessel beam generated by an axicon of opening angle $\gamma$. The superposition of plane waves has wave vectors, which lie on the surface of a cone with opening angle $\theta$.

The finite distance after the axicon in which a BG beam is found is determined from the wave vectors of the Bessel beam that propagate on a cone. This maximum distance is given by,

$$
z_{\max }=\frac{k}{k_{r}} \omega_{0} \approx \frac{\omega_{0}}{\theta}
$$

Within this finite propagation distance, the properties associated with the ideal Bessel beam hold true for a BG beam. Using geometric optics, further information about the beam can be revealed. The maximum width of the BG beam, $r_{\max }$, is found at $z_{\max } / 2$ :

$$
r_{\max }=z_{\max } \tan \theta
$$

These two parameters together with the Bessel function parameter, $k_{r}=k \sin \theta$, allow the number of rings, $N$, at $r_{\max }$ to be solved:

$$
N=\frac{r_{\max }}{2} \frac{1}{\delta}
$$

where $\delta \approx \pi / k_{r}$ is the spacing between each ring.

For such beams, there is a minimum distance behind an obstacle of radius $1 / 2 D$ before reconstruction occurs,

$$
z_{\min }=D \frac{k_{z}}{2 k_{r}}=\frac{D}{2 \tan \theta},
$$

We are able to verify this equation using our conical wave method. Although all the key aspects required to implement this technique are well known, previous models persisted with lengthy integral calculations. 


\section{GEOMETRICAL MODEL}

The model presented here is based on a geometrical approximation to the propagation of conical waves after obstacles. As we will show later, this approximation is amazingly resilient even when the obstacle sizes are in the order of the wavelength of the illuminating light, and so while our method cannot be considered a complete substitute for a full vector diffraction based calculation, it offers a very accurate first approximation.

Consider the schematic in Fig. 3 where an arbitrary obstacle is placed off-centre in the path of a Bessel-Gauss beam. The obstruction cross-section due to this obstacle along one azimuthal direction is shown. The positive $\mathrm{z}$-axis defines the direction of propagation of the Bessel-Gauss beam, which we assume to have a spatial extent of $2 d$, as shown in Fig. 2. Since only a cross-section is shown, there are only two conical waves (labelled $\mathrm{CW}_{1}$ and $\mathrm{CW}_{2}$ respectively) contributing to the Bessel-Gauss beam at any given point.

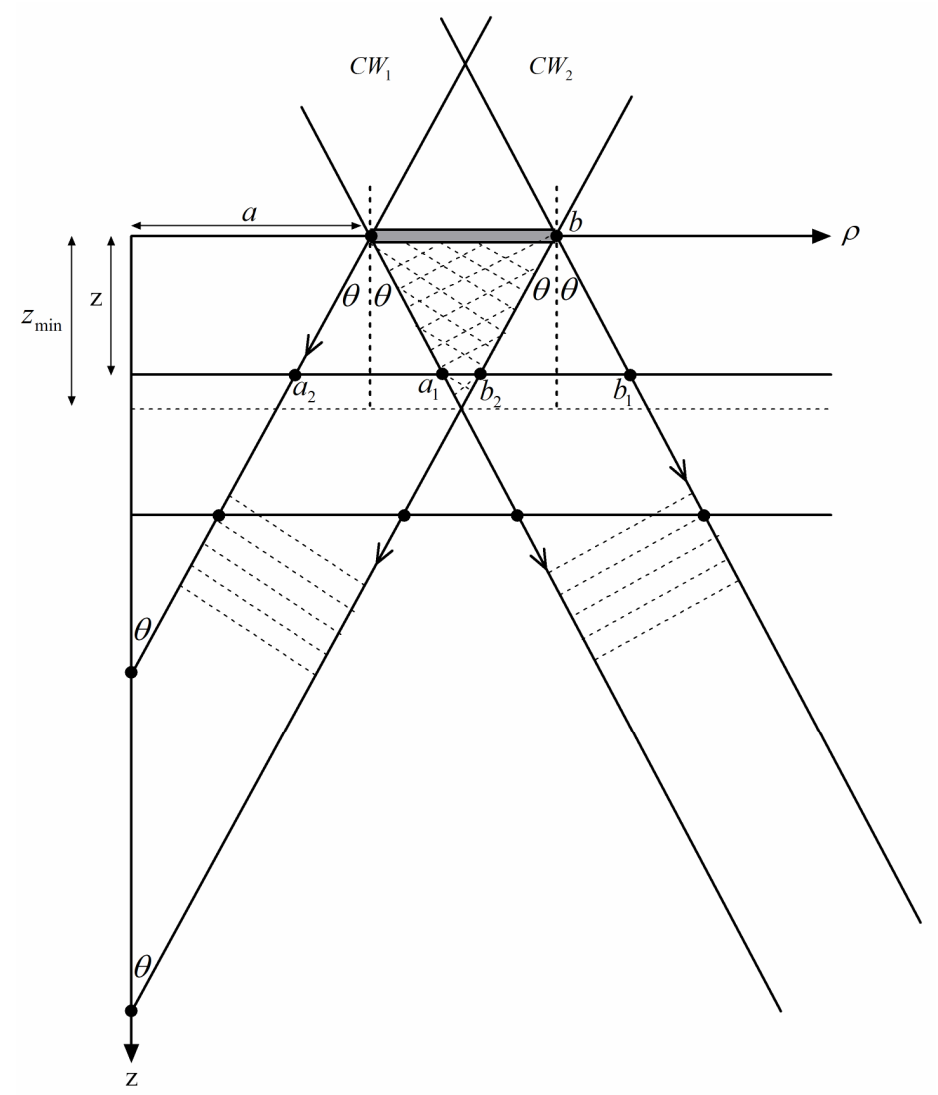

Fig. 3: Diagram illustrating the projection of the obstacle in space by two conical waves, $\mathrm{CW}_{1}$ and $\mathrm{CW}_{2}$. The shadow region where neither conical wave contributes has a minimum distance given by $\mathrm{z}_{\min }$.

It is clear from Fig. 3 that the projection from these two conical waves results in distinct regions defined by (i) one conical wave contribution only, (ii) a shadow region where neither conical wave contributes, and (iii) the Bessel-Gauss beam where both waves contribute. The boundaries that define these regions are mapped by the projection of the obstacle's boundaries along a given $z$ plane. Without any loss of generality, consider an obstacle with cross-section shown in Fig. 3 with full width $D$ and that is placed some distance $\rho=a$ from the central axis $(\rho=0)$ of the BesselGauss beam, so that the two extreme edges of the obstacle are defined by $\rho=a$ and $\rho=b$, with $D=b-a$. From the geometry one can derive the projection of the obstruction due to each conical wave. If the new positions of these edges due to $\mathrm{CW}_{1}$ are labelled $a_{1}$ and $b_{1}$, and similarly $a_{2}$ and $b_{2}$ due to $\mathrm{CW}_{2}$, then one can show that at some distance $z$ after the obstacle we have: 


$$
\begin{aligned}
& a_{1}=a+z \tan \theta \\
& a_{2}=a-z \tan \theta \\
& b_{1}=b+z \tan \theta \\
& b_{2}=b-z \tan \theta
\end{aligned}
$$

Clearly the projection results in the creation of two zones defined by a single conical wave, with the boundaries of these zones moving farther apart at a rate of $2 z \tan \theta$. The region behind the obstacle is then defined as follows (for a given $z$ propagation and a chosen azimuthal angle $\phi$ ):

$-d \leq \rho \leq a_{2} \quad \mathrm{CW}_{1}$ and $\mathrm{CW}_{2}$ add to form a complete Bessel-Gauss beam;

$a_{2}<\rho<a_{1} \quad \mathrm{CW}_{1}$ contribution only;

$a_{1}<\rho<b_{2} \quad$ no contribution from either $\mathrm{CW}_{1}$ or $\mathrm{CW}_{2}$, resulting in a shadow region;

$b_{2}<\rho<b_{1} \quad \mathrm{CW}_{2}$ contribution only;

$b_{1} \leq \rho \leq d \quad \mathrm{CW}_{1}$ and $\mathrm{CW}_{2}$ add to form a complete Bessel-Gauss beam;

Clearly at the distance $z=z_{\min }$ where $b_{2} \leq a_{1}$ the shadow region vanishes and the Bessel-Gauss beam is said to have reconstructed. The value for $z_{\min }$ can easily be found from Eq. (11) by solving for the equality $b_{2}=a_{1}$ :

$$
\begin{aligned}
& b-z \tan \theta=a+z \tan \theta \\
& \Rightarrow b-a=D=2 z \tan \theta \\
& \Rightarrow z_{\min }=\frac{D}{2 \tan \theta}
\end{aligned}
$$

which is consistent with the expression in Eq. (10), as expected. If the process described above is carried out for all points along the boundary of an obstruction, then the complete movement of the boundaries of each region can easily be computed. With this information the complete intensity pattern at any position after an obstacle, regardless of the complexity of the geometry of the obstacle, may readily be found.

It is worth pointing out some salient points in how these regions develop: firstly, the single conical wave regions move apart at a rate of $2 z \tan \theta$. Thus in an off-centred geometry one expects the boundaries defining the Bessel-Gauss beam to move apart, in opposite directions, with one passing through the centre of the original Bessel-Gauss field. Secondly, any combination of points on the boundary of an obstacle that find themselves equidistant from the central propagation axis of the Bessel-Gauss beam will result in boundaries that evolve in identical fashion. This latter fact can be exploited to simplify the calculation for arbitrary obstacles.

\section{EXPERIMENTAL METHODOLOGY AND RESULTS}

Fig. 4 shows the experimental set-up used in generating the Bessel-Gauss beam. A $660 \mathrm{~nm}, 100 \mathrm{~mW}$ diode laser beam was expanded through a $5 \times$ telescope before illuminating an axicon with an opening angle of $\gamma=5^{\circ}$. 


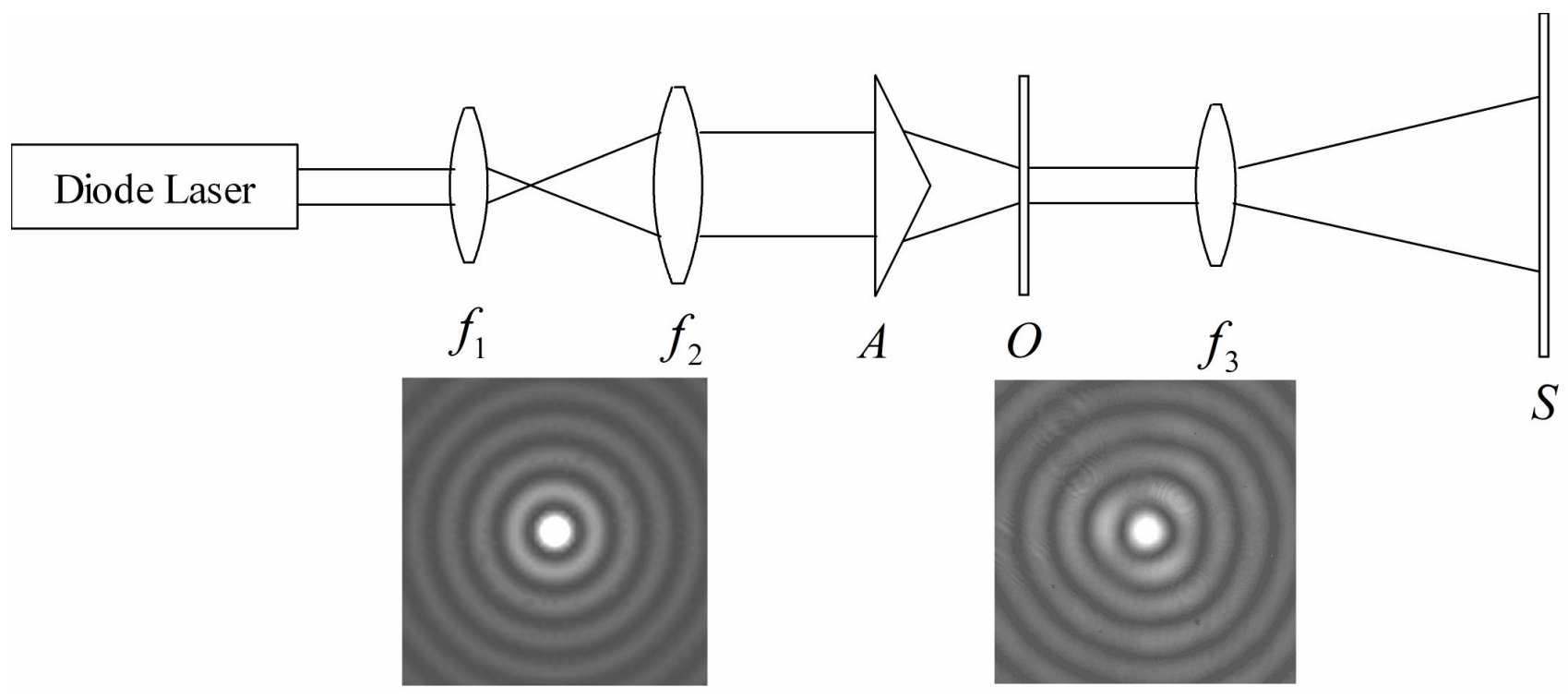

Fig. 4: Experimental design for generating a Bessel-Gauss beam: a telescope with lenses $f_{1}=50 \mathrm{~mm}, f_{2}=250 \mathrm{~mm}$ expands the beam before illuminating the axicon $(\mathrm{A})$. The obstacle $(\mathrm{O})$ was placed $45 \mathrm{~mm}$ from the axicon and was imaged onto a screen $(\mathrm{S})$ by lens $f_{3}$

$=25 \mathrm{~mm}$. The inserts represent the theoretical prediction (left) and the experimentally measured (right) Bessel-Gauss beam.

The obstruction was placed on a thin transparent cover slip and positioned within the Bessel beam at a distance of $1 / 2 z_{\text {max }}$ from the axicon. The resulting obstructed beam was imaged onto a screen with a suitable magnification $(57 \times)$ to observe the intensity variations during propagation. The image plane could be varied continuously by means of a translation stage so as to allow measurement of the beam from fully obstructed to fully reconstructed. The geometries of the two test obstructions - a centred circle (approximately) and a vertical wire - are shown in Fig. 5. In each case the obstruction is opaque so that immediately after the obstruction a dark shadow region is evident, surrounded by an unperturbed Bessel-Gauss beam.

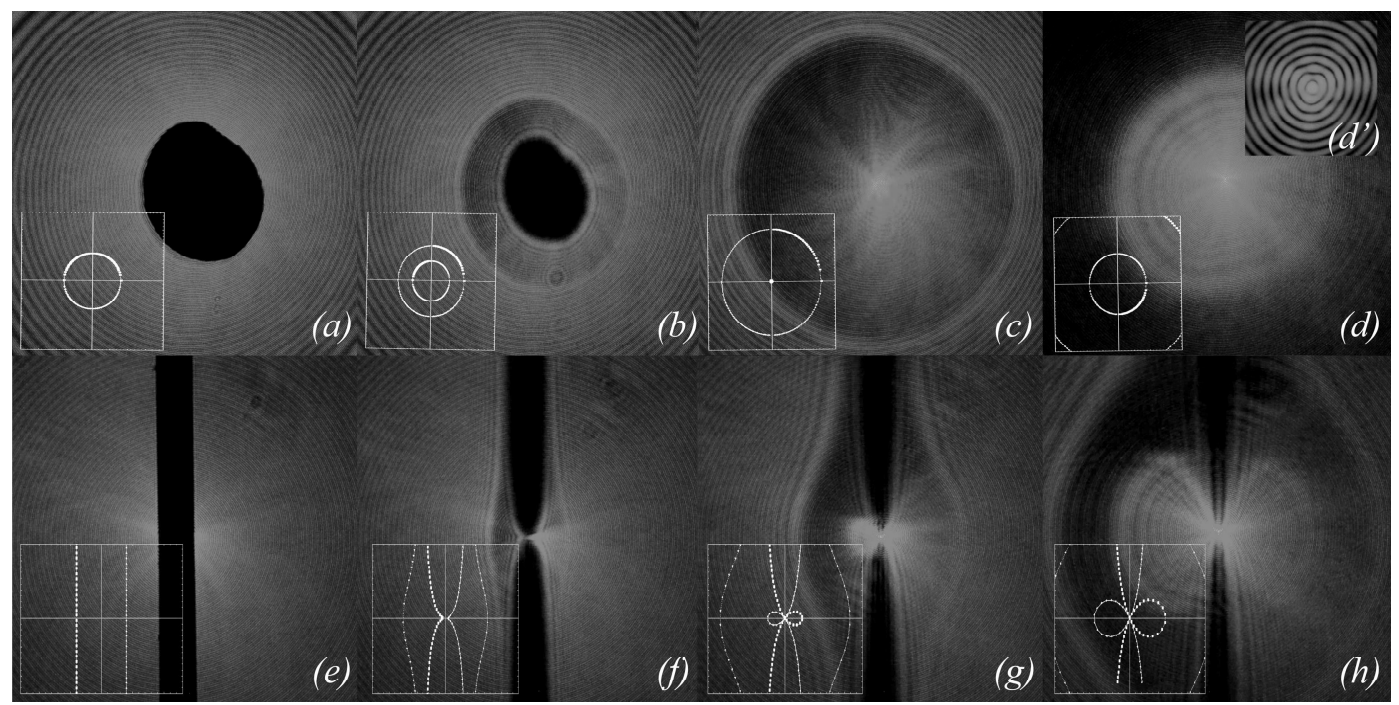

Fig. 5: Experimental images with the theoretical predictions inset in the bottom left corners. The images illustrate the excellent comparison for the self reconstruction of a Bessel beam. 
Fig. 5 shows a sequence of measured Bessel-Gauss beam intensities during propagation after the two obstructions, with the theoretical predictions shown as insets on the experimental images depicting the calculated boundaries due to the conical waves from the source. Figs. 5 (a) - (d) show the change in the Bessel-Gauss beam intensity during reconstruction when the starting obstacle is an approximation to a centred circle, a scenario with radial symmetry. As the propagation distance from the obstruction was increased, so the shadow regions appear to move. Following the discussion in the previous section we note that, as expected, two boundaries appear due to the projection of the obstacle by the two conical waves from the source. The intersection of the two boundaries defines the true shadow region, while the region between the two boundaries defines a single conical wave contribution. Elsewhere, the original BesselGauss beam is evident due to the summation of both conical waves. This is clear in the sequence from (b) to (d). The final image at (d) was taken at a propagation distance that exceeded $z_{\min }$, so that complete reconstruction has taken place. This is predicted by the calculation method and has been confirmed experimentally. Verification of the reconstruction at the centre of the field, where the original obstacle was, is indicated by the zoomed inset of the centre of the beam in (d). A similar sequence is shown in Figs. 5 (e) - (h). In this case the radial symmetry is purposely broken. The sequence illustrates an interesting case with the vertical wire, as here there is a region where no contribution from one of the conical waves is possible, since the obstruction extends across the entire Bessel-Gauss beam in the vertical plane following the wire. Again the agreement between calculation and experiment is excellent.

\section{CONCLUSION}

We have described an effective method to predict the self reconstruction of Bessel and Bessel-Gauss beams after arbitrary obstructions. The model predicts where the beam will reconstruct and also how the shadow regions will progress over the propagation distance. We were able to verify our model experimentally, where the theoretical predictions and experimental results correspond especially well. We have therefore simplified the computations involved in evaluating the self reconstruction of Bessel-Gauss beams, particularly in light of the fact that there are no restrictions on the geometry of the obstacle used, nor its placement in the Bessel field.

\section{REFERENCES}

1. Durnin J., Miceli Jr. J. J. and Eberly J. H., "Diffraction-Free Beams”, Phys. Rev. Lett., 58, 1499, (1987)

2. Durnin J., "Exact solutions for nondiffracting beams. I. The scalar theory", J. Opt. Soc. Am. A, 4, 651, (1986)

3. Gori F., Guattari G. and Padovani C., "Bessel-Gauss Beams", Opt. Commun., 64, 491, (1987)

4. Arlt J., Garces-Chavez V., Sibbett W. and Dholakia K., "Optical micromanipulation using a Bessel light beam", Opt. Commun., 197, 239, (2001)

5. Lu J. and Greenleaf J. F., "Diffraction-limited beams and their applications for ultrasonic imaging and tissue characterization", Proc SPIE, 1733, 92, (1992)

6. Bouchal Z., Wagner J. and Chlup M., "Self-reconstruction of a distorted nondiffracting beam", Opt. Commun., 151, 207, (1998)

7. Sogomonian S., Klewitz S. and Herminghaus S., "Self-reconstruction of a Bessel beam in a nonlinear medium", Opt. Commun., 139, 313, (1997)

8. Bouchal Z. and Bertolotti M., "Self-reconstruction of wave packets due to spatio-temporal couplings", J. Mod. Opt., 47, 1455, (2000)

9. Fischer P., Little H., Smith R. L., Lopez-Mariscal C., Brown C. T. A., Sibbett W. and Dholakia K., "Wavelength dependent propagation and reconstruction of white light Bessel beams", J. Opt. A: Pure Appl. Opt., 8, 477, (2006)

10. Anguiano-Morales M., Mendez-Otero M. and Iturbe-Castillo D., "Conical dynamics of Bessel beams", Opt. Eng., 46(7), 078001-1, (2007)

11. McQueen C. A., Arlt J. and Dholakia K., "An experiment to study a "nondiffracting” light beam", Am. J. Phys. 67, 912, (1999)

12. Stockham A. and Smith J. G., "Optical Design for Generating Bessel Beams for Micromanipulation", Proc. SPIE, 6326, 63261B-1, (2006)

13. Chavez-Cerda S., "A new approach to Bessel beams", J. Mod. Opt., 46, 923, (1999) 